patients, commonly with respect to anticipation of pain or the results of the procedure. The ranked preference scores suggested that interaction with the endoscopist, including technical skill of the endoscopist, discomfort during the procedure, manner of the endoscopist and the pre-and post procedure discussions were considered as most important to patients. A majority of patients (55\%) preferred the endoscopist to explain the findings, but only $26 \%$ specified that they needed to explain the procedure itself. Environmental factors were considered of relatively low importance, including the single sex environment (least important), noise levels, explanation of delay, privacy and intra department waiting time. A majority (82.1\%) thought that having a single sex environment was minimally/not important, and only $14.3 \%$ of patients were prepared to have a delayed appointment for a single sex environment.

Conclusion Patients undergoing colonoscopy appear to highly prioritise aspects of care relating to the interaction with the endoscopist and the procedure itself. Environment factors are considered to have much less value and specifically having a single sex environment. These findings may assist in service redesign around patient-centred care and patients priorities, and the development of patient satisfaction surveys in endoscopy.

Competing interests None declared.

\section{PTU-217 OESOPHAGO-GASTRODUODENOSCOPY YIELD IN PATIENTS WITH COELIAC DISEASE PRESENTING WITH IRON DEFICIENCY ANAEMIA: A RE-AUDIT}

doi:10.1136/gutjnl-2012-302514c.217

K M Khatri, ${ }^{*}$ K Perryman, S Enefer, M Sayegh. General Surgery, Western Sussex Hospitals NHS Trust, Worthing, UK

Introduction In our previous audit it was shown that the majority of patients with iron-deficiency anaemia (IDA) suspected of having coeliac disease (CD) underwent oesophago-gastroduodenoscopy (OGD) and duodenal biopsy as a routine procedure, but only $0.2 \%$ patients had serum coeliac screening prior to OGD. It was suggested that routine duodenal biopsy could be avoided by routine serum coeliac screening, as recommended by the British Society of Gastroenterology (BSG). The purpose of this current study was to complete the audit cycle.

Methods Data related to histology and serum coeliac screen of all patients with IDA undergoing OGD in a District General Hospital from January 1st to October 31st 2011 were evaluated. Data were extracted from Gastrointestinal reporting tool ${ }^{\circledR}$ and analysed in Microsoft Excel ${ }^{\circledR}$ spreadsheet.

Results A total of 732 patients with IDA were referred for OGD. There were 282 male and 450 female patients with a mean age of 69.1 years. Duodenal biopsy was performed in 610 patients (83.3\%) at the time of OGD; CD was confirmed histopathologically in 17 patients (2.8\%). Duodenal biopsy was normal in 593 patients $(97.2 \%)$. A total of 122 patients $(16.7 \%)$ had serum coeliac screening prior to OGD; 7 cases (5.7\%) were positive.

Conclusion Completing the audit cycle it was found that the majority $(83.3 \%)$ of patients with suspected CD presenting with IDA continue to undergo OGD and duodenal biopsy as a routine procedure. CD was confirmed histopathologically in $2.8 \%$ of cases (compared with $2.52 \%$ previously). Of note, $16.7 \%$ of patients had serum coeliac screening prior to OGD, compared with $0.2 \%$ previously. While this represents an improvement in practice the need for wider use of coeliac screening appears to remain

Competing interests None declared.

\section{PTU-218 PILOT RANDOMISED CROSS-OVER STUDY COMPARING THE EFFICACY OF TRANSNASAL ENDOSHEATH ${ }^{\circledR}$ TO STANDARD ENDOSCOPY TO DETECT BARRETT'S OESOPHAGUS}

doi:10.1136/gutjnl-2012-302514c.218

${ }^{1} \mathrm{~K}$ M Shariff, * ${ }^{1} \mathrm{~S}$ Verghese, ${ }^{1} \mathrm{M}$ O’Donovan, ${ }^{1} \mathrm{Z}$ Abdullahi, ${ }^{2} \mathrm{~J}$ Blazeby, ${ }^{1} \mathrm{R} \mathrm{C}$ Fitzgerald. ${ }^{1}$ Hutchison-MRC Research Centre, Cambridge, UK; ${ }^{2}$ Department of Social Medicine, University Bristol, Bristol, UK

Introduction A less expensive and safer alternative to standard sedated endoscopy (SE) needs to be considered as a screening method to detect Barrett's oesophagus (BE) in the population, with the aim of reducing the mortality associated with oesophageal adenocarcinoma. The Endosheath ${ }^{\circledR}$ transnasal oesophagoscope (TNE) can potentially offer a new alternative to conventional standard endoscopy in diagnosing Barrett's oesophagus. The Endosheath ${ }^{\circledR}$ technology uses a sterile, disposable sheath which covers the ultra thin flexible oesophagoscope and isolates it from the patient. The oesophagoscope is placed in a new sheath prior to each procedure which obviates the need for machine washing and permits a quick turnaround. Aim: A pilot study to evaluate the efficacy of TNE in diagnosing BE compared with SE and to assess patient acceptability of TNE.

Methods Patients referred for surveillance endoscopy for BE or a clinically indicated routine endoscopy were recruited to both TNE and $\mathrm{SE}$ in a randomised cross-over design. The interval between the procedures was at least 6 weeks. TNE findings of endoscopic BE, and presence of intestinal metaplasia (IM) on the biopsy samples were compared against SE, which was used as gold standard. A 10-point visual analogue scale ( 0 represented the worst experience and 10 the best experience) to assess the post-endoscopy experience and a single question addressing preference for endoscopy type were used to measure patient acceptability of the procedures.

Results 15 patients completed the study, 10 males and 5 females with a mean age of 62.85 years (range $50-76$ years). Nine of which were BE surveillance patients and six were referred for a clinically indicated routine endoscopy. Eight patients were randomised to the $\mathrm{SE}$ as the first procedure. All the 11 patients with an endoscopic diagnosis of $\mathrm{BE}$ on $\mathrm{SE}$ were accurately identified with the TNE (sensitivity $100 \%$; specificity $100 \%$ ). Biopsies were taken in all the 11 Barrett's segments except in one $<1 \mathrm{~cm}$ segment with TNE due to technical difficulty. IM was detected in 9 out of the 11 patients with BE on SE compared to 7 out of the 11 patients with BE on TNE (sensitivity $77.8 \%$; specificity $100 \%$ ). Patients reported significantly better experiences of endoscopy with TNE with scores of $6.9( \pm 0.81$ SEM) compared with 3.7 ( \pm 0.37 SEM) for SE $(p=0.001)$. Eight patients $(53 \%)$ reported a preference for TNE compared with $1(7 \%)$ for SE.

Conclusion Endosheath ${ }^{\circledR}$ transnasal oesophagoscope is accurate in diagnosing endoscopic BE and can detect IM. It is better tolerated and preferred by patients, making it a useful screening tool for $\mathrm{BE}$ with potential for use in primary care.

Competing interests None declared.

\section{PTU-219 INTRAOPERATIVE ENDOSCOPY: THE FIRST SINGLE-CENTRE UK EXPERIENCE}

doi:10.1136/gutjnl-2012-302514c.219

K Evans, ${ }^{*}$ S Akula, I C Cameron, D S Sanders, M E McAlindon, R Sidhu. Royal Hallamshire Hospital, Sheffield, UK

Introduction Intra-operative enteroscopy (IOE) is the gold standard for examination of the small bowel. However, with the invention of 